EPJ Web of Conferences 80, 00008 (2014)

DOI: $10.1051 /$ epjconf/20148000008

(C) Owned by the authors, published by EDP Sciences, 2014

\title{
Anomalous Transport: Holography, QCD and (Semi-)Metals
}

\author{
Karl Landsteiner ${ }^{1, a}$ \\ ${ }^{1}$ Instituto de Física Teórica UAM/CSIC, Universidad Autónoma de Madrid, C/ Nicolas Cabrera 13-15, 28049 \\ Cantoblanco, SPAIN
}

\begin{abstract}
At finite temperature and density chiral anomalies give rise to dissipationless transport phenomena. The most prominent example being the chiral magnetic effect (CME) and the chiral vortical effect (CVE). I will discuss the theory of anomalous transport from the field theory and holography perspective and comment on applications to the physics of the quark gluon plasma and new, so far hypothetical materials, the Weyl semi-metals.
\end{abstract}

\section{Introduction}

Chiral anomalies are normally thought of as belonging to the realm of elementary particle physics. They have been discovered in the analysis of the triangle Feynman diagram with three chiral currents on its edges and found an immediate and beautiful application in the explanation of the decay of the neutral pion into two photons (see $[1,2]$ for reviews).

The high energy physicist is probably less aware of the important role anomalies play in modern condensed matter physics. In fact the (integer) quantum hall effect can be described in quantum field theory terms via anomaly cancellation on a lower-dimensional defect or boundary via charge inflow (anomaly inflow) from a gapped bulk. Here it is important that there are no low-energy degrees of freedom in the bulk, and all the (chiral) low energy fermions are concentrated on the defect, as is the case e.g. for topological insulators (see [3] for a recent theoretical review).

More recently the focus has been however on anomaly induced transport in systems with chiral bulk fermions. Although one might say that the study of effects of anomalies is always interesting and does not need specific motivation, I think it is fair to say that much of the recent developments in this field have its root in the discovery of the quark gluon plasma that is formed in heavy ion collisions performed at the relativistic heavy ion collider (RHIC) and at the large hadron collider (LHC) in Geneva. In particular anomaly induced charge separation via the chiral magnetic effect has been predicted $[4,5]$ and possibly also observed $[6,7]$. More generally the strongly coupled nature and the associated low shear viscosity to entropy ratio $[8,9]$ have triggered a large amount of research in holographic models of the quark gluon plasma. Such models have lead to a veritable revolution in our understanding of relativistic hydrodynamics and one of the outcomes was the discovery of another anomaly induced transport effect, the chiral vortical effect [10-12].

I will briefly review the theory of these transport phenomena and mention applications to QCD and the physics of heavy ion collisions on the one hand and to modern condensed matter physics and Weyl semi-metals on the other hand.

\footnotetext{
a e-mail: karl.landsteiner@uam.es
} 


\section{Kubo formulas for anomalous transport}

Since these effects describe transport phenomena there are transport coefficients associated to them and these can be computed via suitable Kubo formulas, i.e. two point correlation functions of conserved currents in specific kinematic limits [13-15]. In order to derive the Kubo formula for the chiral magnetic effect we simply write

$$
\vec{J}=\sigma^{B} \vec{B}
$$

This encodes the amount of current generated by an external magnetic field into a conductivity type constant, the chiral magnetic conductivity $\sigma^{B}$. Similarly we write

$$
\vec{J}=2 \sigma^{V} \vec{\omega}
$$

for the chiral vortical conductivity, where $\vec{\omega}=\frac{1}{2} \nabla \times \vec{v}$ is the vorticity. The magnetic field is given as the curl of the vector potential $\vec{B}=\nabla \times \vec{A}$. Since $\vec{A}$ can be viewed as the source for insertions of the current $J$ in correlation functions we get the Kubo formula for the chiral magnetic conductivity by differentiation with respect to the vector potential as

$$
\left\langle J_{k} J_{m}\right\rangle=i \sigma^{B} \epsilon_{k l m} p_{l}
$$

where the dots denote higher order terms in momentum and frequency.

In order to get a Kubo formula for the vortical conductivity we recall the formalism of gravitoelectromagnetism. The off-diagonal components of the metric induce rotation via frame dragging. Therefore we can substitute the vorticity by the gravito-magnetic field $\vec{B}_{g}=\nabla \times \vec{A}_{g}$ where the components of the gravito-magnetic potential are given by the mixed time-space components of the metric $A_{k}=g_{0 k}$. In terms of the gravito-magnetic field the chiral vortical effect can be written as

$$
\vec{J}=\sigma^{V} \vec{B}_{g}
$$

The factor of two is absorbed into the relation between rotation $\vec{\omega}$ and gravito-magnetic potential $g_{0 k}$. Since the metric couples to the energy-momentum tensor we find the Kubo formula as before by differentiating with respect to the metric

$$
\left\langle J_{k} T_{0 m}\right\rangle=i \sigma^{V} \epsilon_{k l m} p_{l} .
$$

Finally we notice that similar formulas hold for the corresponding energy transport and give chiral magnetic and chiral vortical conductivities for the energy current $J_{\epsilon, k}=T_{0 k}$

$$
\left\langle T_{0 k} J_{m}\right\rangle=i \sigma_{\epsilon}^{B} \epsilon_{k l m} p_{l},
$$

and

$$
\left\langle T_{0 k} T_{0 m}\right\rangle=i \sigma_{\epsilon}^{V} \epsilon_{k l m} p_{l},
$$

We finally note that the CME and the CVE are both time-reversal even. In particular this implies that they do not produce any dissipation, i.e. they do not contribute to entropy production.

We also note that Kubo formulas can also be used to study the momentum and frequency dependence of these effects [16]. 

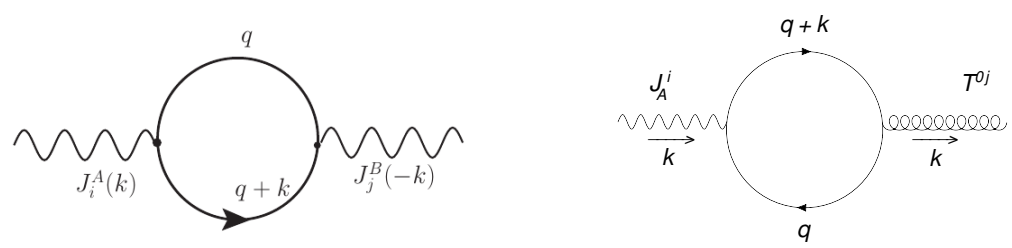

Figure 1. Feynman diagrams corresponding to the Kubo formulas for chiral magnetic and chiral vortical conductivity.

\subsection{Weak coupling}

The simplest system in which above Kubo formula can be evaluated is a gas of free chiral Weyl fermions. In fact one can be a bit more general and allow for a generic symmetry group, generated by $T_{A}$ and compute the response to generalized magnetic fields with indices $A$. One has to study the diagrams in 1 at finite temperature and with chemical potential $\mu_{A}$, where $A$ has to correspond to a generator in the Cartan sub-algebra of the Lie algebra generated by $T_{A}$.

Evaluating these diagrams one finds

$$
\begin{gathered}
\sigma_{A B}^{B}=\frac{d_{A B C}}{4 \pi^{2}} \mu_{C}, \quad \sigma_{A}^{V}=\frac{d_{A B C}}{8 \pi^{2}} \mu_{B} \mu_{C}+\frac{b_{A}}{24} T^{2} \\
\sigma_{\epsilon, A}^{B}=\frac{d_{A B C}}{8 \pi^{2}} \mu_{B} \mu_{C}+\frac{b_{A}}{24} T^{2}, \quad \sigma_{\epsilon}^{V}=\frac{d_{A B C}}{12 \pi^{2}} \mu_{A} \mu_{B} \mu_{C}+\frac{b_{A}}{12} \mu_{A} T^{2}
\end{gathered}
$$

The group theoretical factors are

$$
\begin{aligned}
d_{A B C} & =\operatorname{str}\left(T_{A} T_{B} T_{C}\right)_{L}-\operatorname{str}\left(T_{A} T_{B} T_{C}\right)_{R} \\
b_{A} & =\operatorname{tr}\left(T_{A}\right)_{L}-\operatorname{tr}\left(T_{A}\right)_{R} .
\end{aligned}
$$

These group theoretical factors indeed determine if the fermion spectrum features chiral anomalies. A chiral anomaly is present in the triangle diagram with three currents if and only if $d_{A B C}$ is nonzero. Similarly a mixed gauge-gravitational anomaly is present in the triangle with one current and two energy-momentum tensors if and only if $b_{A}$ is different from zero. Indeed the anomaly as the non-conservation of a current in external background fields can be written as

$$
\left(D_{\mu} J^{\mu}\right)_{A}=\frac{d_{A B C}}{32 \pi^{2}} \epsilon^{\mu v \rho \lambda} F_{\mu \nu}^{B} F_{\rho \lambda}^{C}+\frac{b_{A}}{768 \pi^{2}} \epsilon^{\mu \nu \rho \lambda} R_{\beta \mu \nu}^{\alpha} R_{\alpha \rho \lambda}^{\beta} .
$$

\subsection{Strong coupling}

A rather successful approach to study strongly coupled relativistic plasma is the AdS/CFT correspondence [17]. It is known to give realistic results for transport coefficients such as the shear viscosity. If we apply this approach to the study of anomaly related transport we do expect however exact results since anomalies are known to be subject to non-renormalization theorems (see figure 2). The simplest AdS/CFT model that contains both, chiral and mixed anomalies is given by the action [18]

$$
\begin{aligned}
S= & \frac{1}{16 \pi G} \int_{M} d^{5} x \sqrt{-g}\left[R+2 \Lambda-\frac{1}{4} F_{M N} F^{M N}\right. \\
& \left.+\epsilon^{M N P Q R} A_{M}\left(\frac{\kappa}{3} F_{N P} F_{Q R}+\lambda R_{B N P}^{A} R_{A Q R}^{B}\right)\right]+S_{G H}+S_{C S K},
\end{aligned}
$$




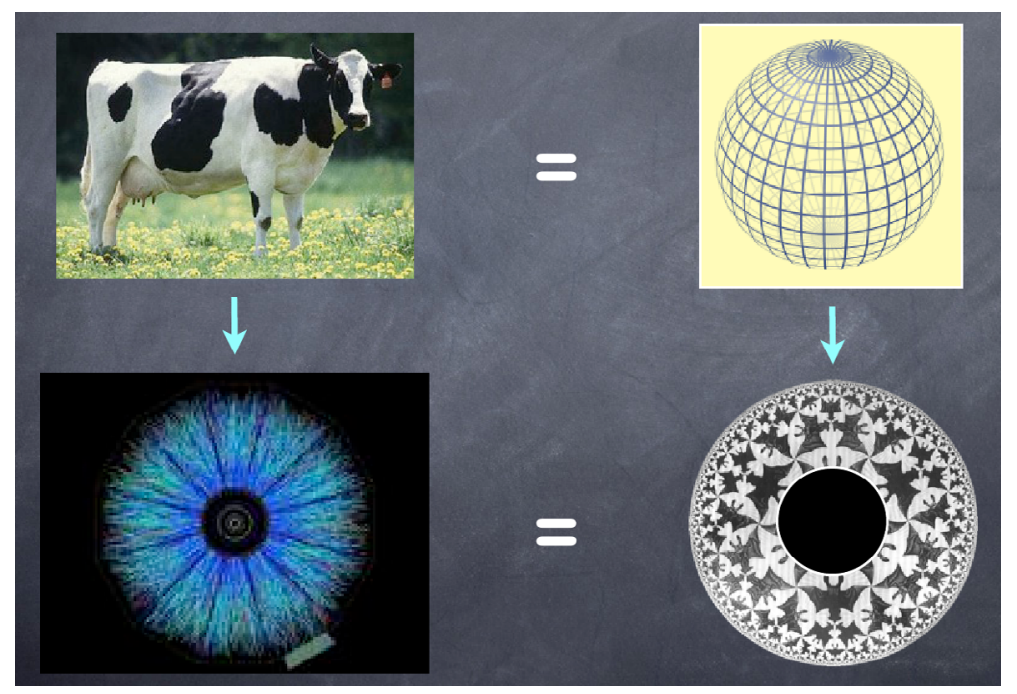

Figure 2. The AdS/CFT correspondence is often said to provide the "spherical cow" toy model for the strongly coupled quark gluon plasma. If we are interested in observables related to topology, such as anomalies are generically, a spherical cow toy model should however be sufficient to obtain exact results.

$$
\begin{aligned}
S_{G H} & =\frac{1}{8 \pi G} \int_{\partial M} d^{4} x \sqrt{-h} K, \\
S_{C S K} & =-\frac{1}{2 \pi G} \int_{\partial M} d^{4} x \sqrt{-h} \lambda n_{M} \epsilon^{M N P Q R} A_{N} K_{P L} D_{Q} K_{R}^{L},
\end{aligned}
$$

Based on this model one can compute the Kubo formulas in holography and without going into the details let me just state that one finds a result that is in complete agreement with the weak coupling results (8)-(9). This gives a good indication for a non-renormalization theorem to be at work also for the chiral conductivities. In fact, the non-renormalization of the terms depending only on the chemical potentials can be inferred from hydrodynamics alone. The arguments for non-renormalization of the temperature dependent terms are more involved, but by now they are also well-established in different approaches [19-21].

\section{The consistent and covariant currents}

So far we have discussed anomalous transport without really specifying the type of current we are using. Anomalies can be viewed as consistent anomalies, i.e. fulfilling the Wess-Zumino consistency condition. Alternatively they are often expressed in terms of the covariant anomaly which transforms covariantly under the gauge transformations, even the anomalous ones [22]. Correspondingly there are two commonly used notions of currents, the covariant current and the consistent current. The divergence of the covariant current gives the covariant anomaly and the divergence of the consistent current gives the consistent current. Both are related by the so-called Bardeen-Zumino polynomials. In the case of a simple V-A model with axial and vector-like $U(1)$ symmetry the covariant and consistent vector-like currents are related by

$$
J_{\text {cons }}^{\mu}=J_{\text {cov }}^{\mu}+\frac{1}{4 \pi^{2}} \epsilon^{\mu \nu \rho \lambda} A_{v}^{5} F_{\rho \lambda} .
$$


Here $A^{5}$ is an axial gauge potential that couples to the axial current $J_{5}^{\mu}$. We note that only the consistent current is conserved even in the presence of an axial gauge field $\partial_{\mu} J_{\text {cons }}^{\mu}=0$ whereas the covariant current obeys $\partial_{\mu} J_{\text {cov }}^{\mu}=\frac{1}{8 \pi^{2}} \epsilon^{\mu \nu \rho \lambda} F_{\mu \nu} F_{\rho \lambda}^{5}$. Keeping the notion of axial chemical potential and axial gauge field apart we have for the $\mathrm{CME}$ in the consistent current therefore the result

$$
\vec{J}_{\text {cons }}=\frac{1}{2 \pi^{2}}\left(\mu_{5}-A_{0}^{5}\right) \vec{B}
$$

where $2 \mu_{5}=\mu_{L}-\mu_{R}$ is the axial chemical potential. In particular if we identify $\mu_{5}=A_{0}^{5}$ we find a vanishing CME. We will give an interpretation of this in the following in a possible application to Weyl semi-metals.

\section{Applications}

\subsection{Heavy ion collisions}

In heavy ion collisions a chiral imbalance (difference in the numbers of left-handed and right-handed fermions), i.e. an axial chemical potential, can be generated by exciting topologically non-trivial gluon gauge field configurations in the early non-equilibrium stages of the collision. The axial anomaly in has indeed two parts, a QCD and a QED part. Once the chiral imbalance is induced via the QCD axial anomaly the CME effect takes place via the QED axial anomaly. The magnetic field in generated by the by-flying spectators, i.e. the parts of the nuclei that do not take part in the collision. This situation is much more complicated that what we considered in our simple theory outline. The magnetic field exists only for a short time span and the non-renormalization theorems are known not to hold when dynamical gauge field [19, 23, 24] (such as the gluon field of QCD) are present in contrast to external classical fields we have considered up to now. A holographic toy model and a first study of anomaly related transport in such a situation has recently been performed in [25, 26]. Another point is somewhat clearer: there is no evidence for an axial gauge potential in nature. Because of the axial anomaly such gauge potential would be an observable in contrast the true, vector-like gauge potential. Therefore we can set the second term in (17) to zero. We expect the CME to be realized in the plasma phase of QCD and this is indeed compatible with observational facts inferred from heavy ion collisions.

\subsection{Weyl semi-metals}

Weyl semi-metals can be understood as a sort of three dimensional generalization of Graphene (see [27] for a recent review). In the simplest model there are two bands, a conduction band and a valence band. These two bands touch however at two isolated points in the Brillouin zone such that the electronic structure is described by the following diagram

The separation in (pseudo)momentum and frequency of the two Weyl nodes of opposite chirality can be modeled as the presence of an axial gauge potential $A_{\mu}^{5}$. The chemical potentials are measured from the tips of the cones, i.e. the usual normal ordered vacuum of quantum field theory. Then it is easy to see that in an equilibrium situation in which the Fermi levels of the left- and right-handed Weyl cones coincide we have precisely $\mu_{5}=A_{\mu}^{5}$ and the cancellation present in (17) takes place and the CME is not effective in the equilibrium configuration of the WSM. En-passent we note that the spatial part of the axial vector potential gives rise to the quantum anomalous Hall effect in the consistent current (16)

$$
\vec{J}=\frac{1}{2 \pi^{2}} \overrightarrow{A_{5}} \times \vec{E}
$$




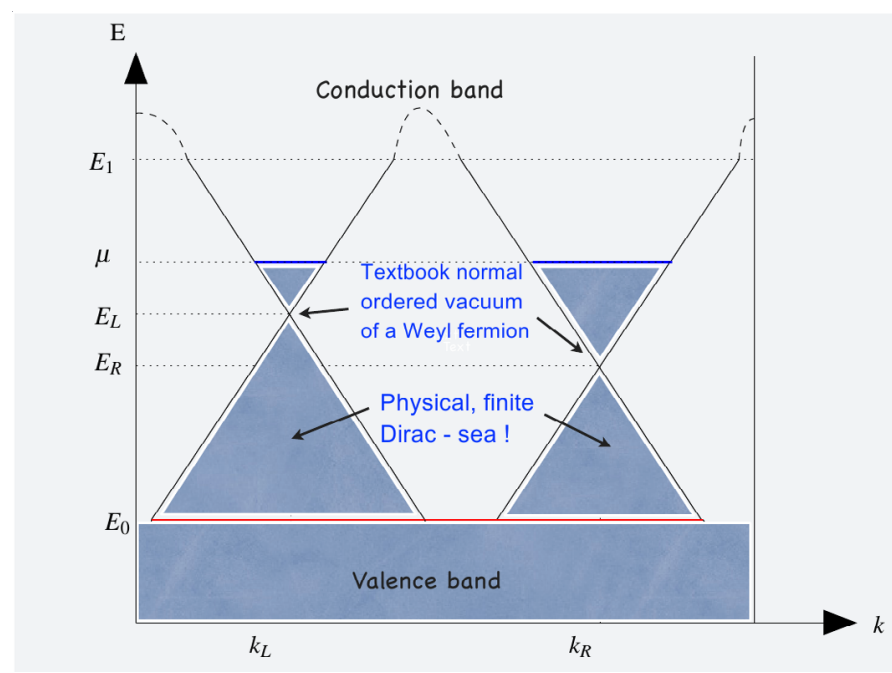

Figure 3. Sketch of the Brillouin zone of a Weyl (semi)-metal. Valence and conduction bands touch in two points. The local Hamiltonian describing the dynamics of the electrons near the band touching points is the one of a left-handed and right-handed Weyl fermion. The band touching points can sit however at different points in the Brillouin zone and also at different energies. One approach to the physics of WSMs takes this separation as a background for the axial vector potential $1 / 2\left(E_{L}-E_{R}, \vec{k}_{L}=\vec{k}_{R}\right)=A_{\mu}^{5}$.

in and electric field.

Further applications of the theory of anomalous transport to WSMs include the so-called axial magnetic effect [28]. This describes the generation of an energy current in the presence of an axial magnetic field. This is a magnetic field that couples to left- and right-handed Weyl-fermions with opposite signs.

$$
\vec{J}_{\epsilon}=\frac{1}{12} T^{2} \vec{B}_{5}
$$

Since in WSMs such fields can be effectively modeled via the separation of the the Weyl-nodes in the Brillouin zone it has been suggested that this effect might be observable in WSMs. One exciting perspective on this is that the temperature dependence has a simple quadratic form and is a direct consequence of the presence of a mixed axial-gravitational anomaly. Therefore WSMs provide the unique possibility to observe effects of this somewhat exotic anomaly in table top experiments in solid state physics [29].

Acknowledgements I would like to thank all my collaborators on this wonderful and interesting subject for their work and the sharing of their insights with me. This work has been supported by Plan Nacional de Altas Energías FPA2009-07890, Consolider Ingenio 2010 CPAN CSD200-00042 and Severo Ochoa award SEV-2012-0249.

\section{References}

[1] R. A. Bertlmann, Anomalies in quantum field theory Oxford, UK: Clarendon (1996) 566 p. (International series of monographs on physics: 91) 
[2] K. Fujikawa and H. Suzuki, Path integrals and quantum anomalies Oxford, UK: Clarendon (2004) $284 \mathrm{p}$

[3] Shen, Shun-Qing, Topological Insulators: Dirac Equation in Condensed Matters Springer Series in Solid-State Sciences, Vol. 174

[4] D. E. Kharzeev, L. D. McLerran and H. J. Warringa, Nucl. Phys. A 803 (2008) 227 [arXiv:0711.0950 [hep-ph]].

[5] K. Fukushima, D. E. Kharzeev and H. J. Warringa, Phys. Rev. D 78 (2008) 074033 [arXiv:0808.3382 [hep-ph]].

[6] L. Adamczyk et al. [STAR Collaboration], Phys. Rev. Lett. 113 (2014) 052302 [arXiv:1404.1433 [nucl-ex]].

[7] B. Abelev et al. [ALICE Collaboration], Phys. Rev. Lett. 110 (2013) 012301 [arXiv:1207.0900 [nucl-ex]].

[8] E. Shuryak, Prog. Part. Nucl. Phys. 53 (2004) 273 [hep-ph/0312227].

[9] P. Kovtun, D. T. Son and A. O. Starinets, Phys. Rev. Lett. 94 (2005) 111601 [hep-th/0405231].

[10] J. Erdmenger, M. Haack, M. Kaminski and A. Yarom, JHEP 0901 (2009) 055 [arXiv:0809.2488 [hep-th]].

[11] N. Banerjee, J. Bhattacharya, S. Bhattacharyya, S. Dutta, R. Loganayagam and P. Surowka, JHEP 1101 (2011) 094 [arXiv:0809.2596 [hep-th]].

[12] D. T. Son and P. Surowka, Phys. Rev. Lett. 103 (2009) 191601 [arXiv:0906.5044 [hep-th]].

[13] D. E. Kharzeev and H. J. Warringa, Phys. Rev. D 80 (2009) 034028 [arXiv:0907.5007 [hep-ph]].

[14] K. Landsteiner, E. Megias and F. Pena-Benitez, Phys. Rev. Lett. 107 (2011) 021601 [arXiv:1103.5006 [hep-ph]].

[15] K. Landsteiner, E. Megias and F. Pena-Benitez, Lect. Notes Phys. 871 (2013) 433 [arXiv:1207.5808 [hep-th]].

[16] See E. Megias' contribution to this volume.

[17] O. Aharony, S. S. Gubser, J. M. Maldacena, H. Ooguri and Y. Oz, Phys. Rept. 323 (2000) 183 [hep-th/9905111].

[18] K. Landsteiner, E. Megias, L. Melgar and F. Pena-Benitez, JHEP 1109 (2011) 121 [arXiv:1107.0368 [hep-th]].

[19] S. Golkar and D. T. Son, arXiv:1207.5806 [hep-th].

[20] K. Jensen, R. Loganayagam and A. Yarom, JHEP 1302 (2013) 088 [arXiv:1207.5824 [hep-th]].

[21] L. Di Pietro and Z. Komargodski, arXiv:1407.6061 [hep-th].

[22] W. A. Bardeen and B. Zumino, Nucl. Phys. B 244 (1984) 421.

[23] D. F. Hou, H. Liu and H. c. Ren, Phys. Rev. D 86 (2012) 121703 [arXiv:1210.0969 [hep-th]].

[24] K. Jensen, P. Kovtun and A. Ritz, JHEP 1310 (2013) 186 [arXiv:1307.3234 [hep-th]].

[25] A. Jimenez-Alba, K. Landsteiner and L. Melgar, arXiv:1407.8162 [hep-th].

[26] U. Gursoy and A. Jansen, arXiv:1407.3282 [hep-th].

[27] P. Hosur and X. Qi, Comptes Rendus Physique Volume 14, Issues 9-10, November-December 2013, Pages 857-870 [arXiv:1309.4464 [cond-mat.str-el]].

[28] V. Braguta, M. N. Chernodub, K. Landsteiner, M. I. Polikarpov and M. V. Ulybyshev, Phys. Rev. D 88 (2013) 071501 [arXiv:1303.6266 [hep-lat]].

[29] M. N. Chernodub, A. Cortijo, A. G. Grushin, K. Landsteiner and M. A. H. Vozmediano, Phys. Rev. B 89 (2014) 081407 [arXiv:1311.0878 [hep-th]]. 
